\title{
Slope Stability Procedures used in Landslide Evaluations in Practice
}

\author{
Nilmar JANBU*
}

\begin{abstract}
A main objective of this paper is to demonstrate, by examples, the vast applicability of simple, direct formulae and graphs to solve a variety of slope stability problems in practice. The basic developments of these tools took place between 1920 and 1955 , roughly speaking, although a number of refinements have been added since. Only overall average conditions were considered in these approaches. Herein, three alternatives are presented, named Simple Equilibrium Analyses, SEA, Resistance Envelopes, RE, and Stability Charts, SC.

The practical exploration of the approximate stress distribution within a soil mass of a potential slide was based on limit equilibrium principles and the method of slices using non-circular shear surfaces. This approach was called the Generalized Procedure of Slices, GPS, Janbu (1957, 1973, 1996). A spread sheet version of GPS will be presented and used in an analysis of the initial slopes at the Finneidfjord Slide in June 1996. The numerical GPS-results compare well with the SEA, RE and SC analyses.

Keywords : slope stability, internal equilibrium stresses, numerical procedures.
\end{abstract}

\section{Introduction. Basics}

The basic principle of the classical approach to slope stability analyses was to search for the minimum factor of safety with respect to shear failure:

$$
\begin{aligned}
& F=\tau_{f} / \tau, \text { where } \\
& \tau_{f}=\text { shear strength of the subsoil and } \\
& \tau=\text { shear stress required for equilibrium, } \\
& F=\text { safety factor. }
\end{aligned}
$$

For plane strain conditions and fairly regular slopes and boundary conditions the shear surface through the soil profile was chosen as a circle, Hultin (1916), Pettersen (1916) and Fellenius (1918). The minimum $\mathrm{F}$ was obtained by varying the size and the location of the trial circles.

The classical approach used two different assumptions for shear strength

$$
\begin{aligned}
& \tau_{f}=s=s_{u}=\text { constant (total stress) } \\
& \tau_{f}=c+\sigma^{\prime} \tan \phi=\text { (effective stress) }
\end{aligned}
$$

For a given case the numerical calculations required to obtain the minimum $F$ was formidable (to say the least) at a time when the slide rule was the only practical tool available. This was particularly true for the effective stress analyses.

For simple slopes this tedious time-consuming work was reduced to an absolute minimum by Taylor (1937). Taylor presented his solutions in the form of dimensionless diagram, both for constant and linear strength. Janbu (1954) extended the diagrams to include surcharge, external and internal water levels (GW), tension cracks and the center coordinates of the critical circle.

Eq. (1-1) shows that the shear strength of the subsoil had to be known before the conventional analysis could

\footnotetext{
*) Professor Emeritus, NTNU, Trondheim Norway
}

be performed. Since average values of $\tau, \tau_{f}$ and $F$ are used, one can reformulate Eq. (1-1) as follows

$$
\tau_{\max }=\frac{\tau_{f}}{F_{\min }}=\bar{\tau}_{c}
$$

This means that one can seek directly the average shear stress required for equilibrium $\tau_{c}$, without a priori knowledge of strength. In this paper the basic principle will therefore be to search directly for the aver-
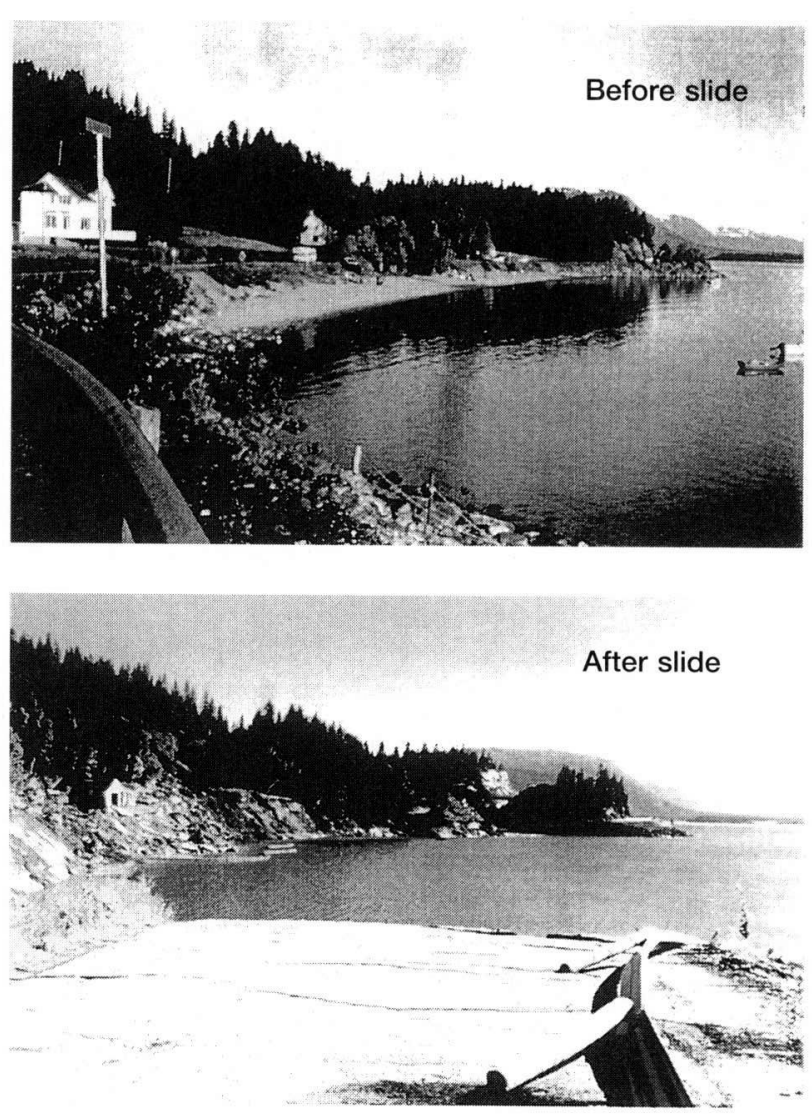

Fig. 1 The slide in Finneidfjord around midnight June 19, 1996 
age equilibrium stress conditions. All that is required is a rewriting of the existing formulae.

\section{The Finneidfjord Slide}

Around midnight on June 19-20, 1996 a landslide occurred in the Finneidfjord area in northern Norway.

Fig. 1 shows a photo of the very beautiful beach area before the landslide, and a photo of the same area a few days after the slide.

During the several weeks that followed a variety of information about the slide was obtained by interviews, eye-witness accounts, bottom search for lost persons and for identifying debris, old and new soil investigation and profiling, and climate statistics, in addition to facts about construction activities along the highway and blasting operations for a new tunnel.

I was asked to evaluate the collected sources of information in order to arrive at an independent evaluation of the possible causes of the slide.

Altogether, about 1 million $\mathrm{m}^{3}$ of soil were involved in the slide; most of it was submerged, see Fig. 2. The slide took four lives, two family houses, $350 \mathrm{~m}$ of the main north-south highway, E6, and some of the tunnelling construction equipment and facilities.

The layered subsoil in the beach area consisted of silt and soft, sensitive clays, with strata of quick clays, under a surficial beach-sand layer. The sand was a few meters thick near shore becoming thinner away from the shoreline. Substantial layers of silt were found particularly in the southern part of the area.

The sequence of sliding has been reconstructed from a careful scrutiny of all information available, particularly from eye witnesses and bottom searches. Initial slides started underwater, before midnight on June 19, at the steepest parts of the slope, some 50-70 m from highway E6, see Fig. 2. Eyewitnesses saw waves, bubbles and whirls at sea level, away from the shore, some time before midnight.

Retrogressive sliding worked its way towards the highway. About 25 minutes after midnight, highway E6 was apparently still in place, according to a car driver, who stopped abruptly, because the car and the highway were shaking strongly. The beach was gone, and the sea made waves close to E6. Minutes later he saw $250 \mathrm{~m}$ of the highway break into three parts and sink into the sea, taking with it a car and its driver (killed). This was followed by movement of the nearest house, with three people, who did not escape. The house first moved straight out then it twisted and tilted backward as it sank into the mud and was pushed into the sea. This part of the slide happened quickly, it was all over in 5 minutes or less.

Several afterslides occurred at the edges of the slide area, taking with them another family house, an excavator, $50 \mathrm{~m}$ of new highway construction, and contractor facilities. In the course of 1 hour, all visible slide activity had stopped. It is the profile in Fig.2 which will be analysed by the various procedures presented below.

A critical search for the cause (s) that triggered the initial slide did not pin-point one single factor. Instead, it is most likely that a combination of several factors, e. g. such as a higher groundwater table due to thawing and periods of rainfall, heavy traffic on a bumpy, rough road, rock blasting, incipient water into surface cracks and into open slits between the steep rock face and the soil, and possibly water-main leakage, all of which could lead to seepage toward the unprotected submarine slope, and even create excess pore pressure.

\section{Simple numerical procedures}

Stability evaluations in practice consist usually of

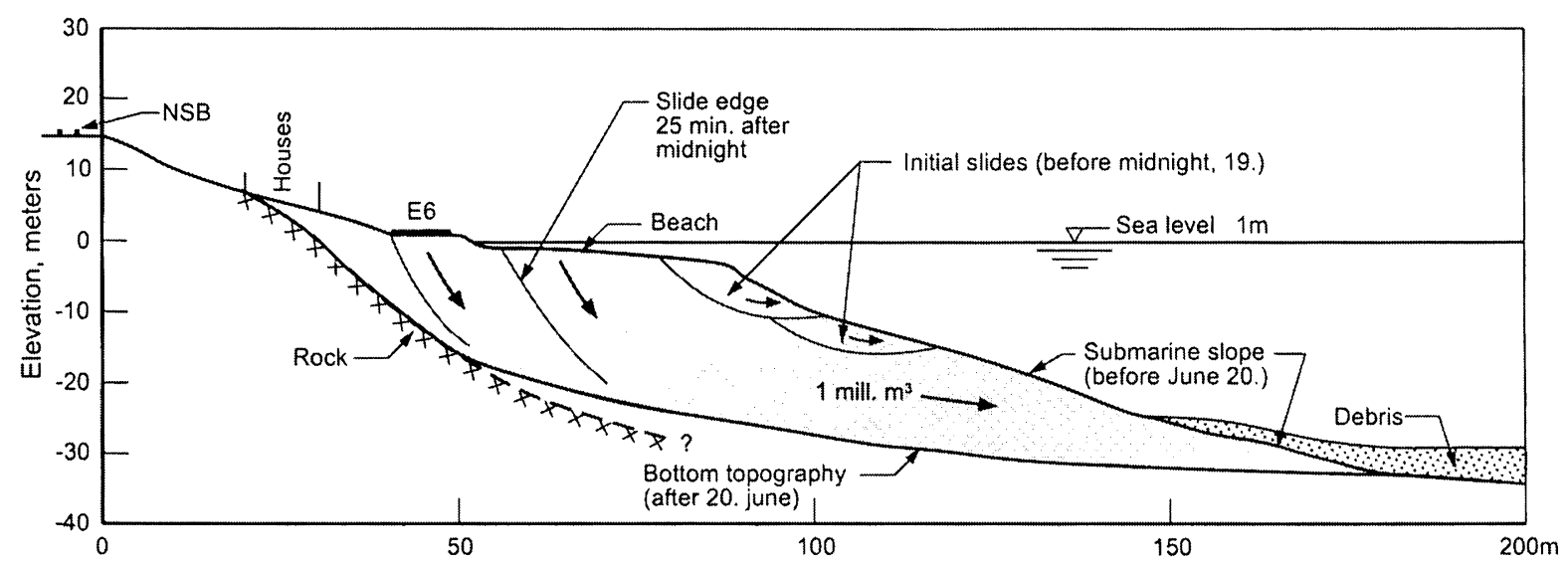

Fig. 2 The central profile of the slide at Finneidfjord 
three entirely different tasks :

- subsoil explorations (experimental)

- equilibrium stress analyses (theory)

- and safety assessment (experience).

Subsoil exploration is by far the most challenging and expensive part, requiring topography, geology, sampling and in situ measurements and laboratory testing, all of which should together lead to relevant soil profiles for stability evaluations.

By contrast, the overall equilibrium stress analyses can in most cases be carried out in simple inexpensive ways, which are the main scope of this paper. Several alternatives will be presented.

\subsection{Simple equilibrium analyses, SEA}

One can seek directly the average shear stress required for equilibrium, without a priori knowledge of strength. For example the classical solution for circular surfaces and simple slopes $\left(F=N_{0} s / \gamma H\right)$ will in equilibrium form $(\tau=s / F)$ read

$$
\bar{\tau}=\frac{\gamma H}{N_{\tau}}
$$

where the approximate equilibrium shear stress number $N_{\tau}=N_{0}$ is shown in Fig. 3 as function of the slope ratio $b=\cot$. $\beta$. If the undrained shear strength is $s_{u}$, the factor of safety becomes

$$
F_{u}=\frac{s_{u}}{\bar{\tau}}
$$

Fig. 3 also contains the corresponding equilibrium normal stress number $N_{o}$ for calculation of the average total normal stress $\sigma$ along the critical toe circle

$$
\bar{\sigma}=\frac{\gamma H}{N_{\sigma}}
$$

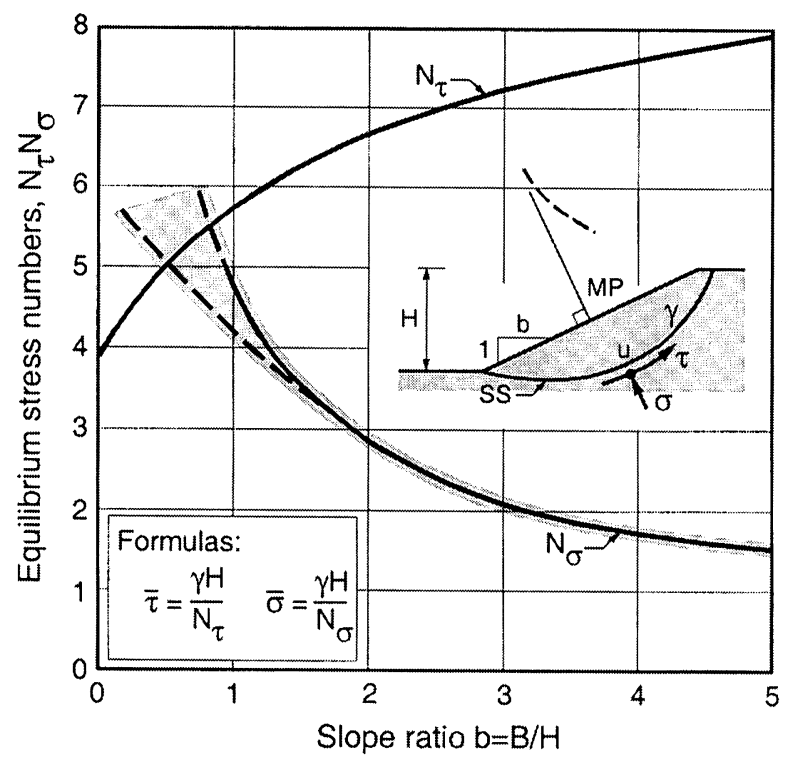

Fig. 3 Simple limit equilibrium analyses, SEA
The evaluation of $N_{\tau}$ and $N_{\sigma}$ was obtained from Janbu (1954).

In terms of effective stress the shear strength is expressed as

$$
\tau_{f}=\left(\sigma^{\prime}+a\right) \tan \phi
$$

where attraction $a=c \cdot \cot \phi$. Hence, the average equilibrium stress

$$
\bar{\tau}=\left(\overline{\sigma^{\prime}}+a\right) \tan \bar{\rho}
$$

where $\tan \rho=\tan \phi / F$ average friction, mobilized at equilibrium. Therefore, when $\tau$ and $\overline{\sigma^{\prime}}$ are calculated the mobilized friction can be esti-mated

$$
\tan \bar{\rho}=\frac{\bar{\tau}}{a^{\prime}+\overline{\sigma^{\prime}}}
$$

The average effective stress $\overline{\sigma^{\prime}}$ is often expressed as

$$
\overline{\sigma^{\prime}}=\left(1-r_{u}\right) \bar{\sigma}
$$

where $r_{u}=$ pore pressure ratio, $u / \sigma$, in general.

The average factor of safety in effective stress then becomes

$$
F_{c}=\frac{\tan \phi}{\tan \bar{\rho}}
$$

where $\tan \phi=$ internal friction in Eq. (31-4).

\subsection{Resistance Envelope procedure, RE}

About 50 years ago one was able to explore the average equilibrium state of stress behind a slope independent of a priori knowledge of shear strength. The procedure developed at Harvard University is known as the resistance envelope procedure (Janbu 1954).

The 1954-resistance envelope diagram for simple slopes is shown in Fig. 4. This diagram will be used for

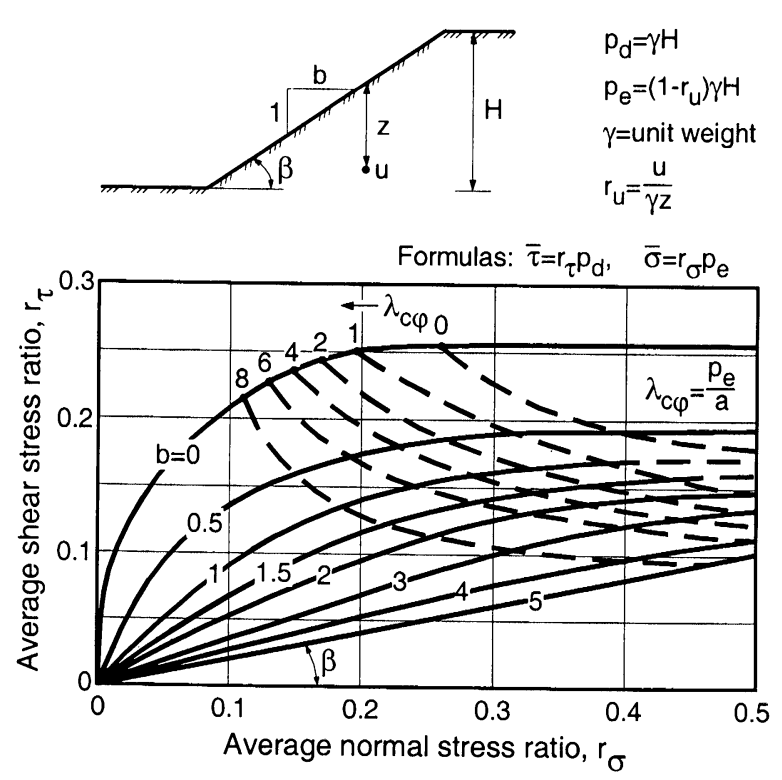

Fig. 4 Resistance envelopes, RE, 1954 
the analyses of the (central) slope at Finneidfjord, see pt. 3.4 .

\subsection{Stability charts for simple slopes}

Stability charts for total stress analyses of simple slopes is shown in Fig. 5A. The original diagram from 1954 was used to find directly the minimum safety factor. The coordinates of the center of the critical circle was expressed as

$$
X_{0}=x_{0} H, \quad Y_{0}=y_{0} H
$$

The dimensionless numbers, $N_{0}, x_{0}$ and $y_{0}$ are plotted as function of the slope ratio $b=\cot \beta$ and the location of rock, or hard layers, $D=d H$ in Fig. 5A.

First of all one gets the maximum average shear stress $\bar{\tau}=s / F_{\min }$ required for equilibrium, from the equation

$$
\begin{aligned}
& \bar{\tau}_{\max }=\frac{\gamma H}{N_{0}} \\
& \text { after which } F_{u}=s_{u} / \bar{\tau}_{\text {max }}
\end{aligned}
$$

Stability charts for effective stress analyses are shown in Fig. 5B. The conventional use of this chart was to obtain the minimum safety

$$
F_{\min }=N_{c f} \frac{c}{P_{d}}
$$

for linear strength $\tau_{f}=c+\sigma^{\prime} \tan \phi$. For simple slopes

$$
p_{d}=\gamma H \text { or }\left(\gamma H-\gamma_{w} H_{w}\right)
$$

In 1954 the critical stability number $N_{c f}$ was found to be a function of the slope ratio $b$ and parameter

$$
\lambda_{c \phi}=\left(\frac{P_{e} \tan \phi}{c}\right)=\frac{P_{e}}{a}
$$

where $p_{e}=\left(1-r_{u}\right) p_{d}$, and $r_{u}=$ (excess) pore pressure ratio. Most often toe circles were found to be critical.

The center coordinates $\left(x_{0} y_{0}\right)$ of the critical toe circles are also shown in Fig. 5B.

Since $c=a \tan \phi$ and $\tan \rho=\tan \phi / F$ one can solve Eq. (33-3) with respect to the average, maximum equilibrium friction, hence

$$
\tan \bar{\rho}=\frac{P_{d}}{a N_{c f}}
$$

after which $F_{e}=\tan \phi / \tan \rho$.

\subsection{Numerical examples}

The most critical parts of the variable slope at Finneidfjord had an inclination around $1: 2$, ie $b=2$, and a height of $\mathrm{H} \sim 10 \mathrm{~m}$, totally submerged with a unit weight $\gamma^{\prime} \approx 9 \mathrm{kN} / \mathrm{m}^{3}$. These data will be chosen for the numerical analyses. The average effective stress parameters were found to be

$a=5 \mathrm{kPa}, \tan \phi=0.45$ with substantial scatter. The analyses also include the possibility of excess pore pressure, with $r_{n} \cong 0.1$.

Using the SEA diagram in Fig. 3 one finds

$$
N_{\tau}=6.7 \text { and } N_{\sigma}=2.9
$$

and hence, since $\gamma^{\prime} H=90 \mathrm{kPa}$,

$$
\tau=13.4 \mathrm{kPa}, \sigma^{\prime}=31.0 \mathrm{kPa}
$$

With $s_{u}=10-15 \mathrm{kPa}$ the undrained safety $F_{u}$ is around $1.0 \pm$.

For zero attraction $a=0$ (longterm)

$$
\tan \bar{\rho}=\frac{\bar{\tau}}{\overline{\sigma^{\prime}}}=0.43
$$

and the corresponding effective stress safety is

$$
F_{c}=0.45 / 0.43=1.04
$$

hence very low.

For $r_{u}=0.1$ the effective stress $\sigma^{\prime}=(0.9 \bullet 31)=27.9 \mathrm{kPa}$ leading to

$$
\tan \rho=13.4 / 27.9=0.48
$$

in which case $F_{e}=0.94$ for zero attraction. An attraction of $2 \mathrm{kPa}$ would bring $F_{e}$ to 1.0 . This example goes to show how sensitive the slope stability is to excess pore pressure and loss of attraction (over time).

Using the RE-diagram in Fig. 4, one selects increasing values of

$$
r_{\sigma}=0-0.1-0.2-0.3
$$

and obtains the corresponding

$$
r_{\tau}=0-0.05-0.09-0.12
$$

for $b=2$. Then one calculates

$$
\tau=r_{\tau} p_{d}, \quad \sigma^{\prime}=r_{\sigma} p_{e}
$$

for each $r_{\tau}, r_{\sigma}$ selection. With $r_{u}=0$ and $p_{d}=p_{e}=90 \mathrm{kpa}$, one obtains

$$
\begin{aligned}
& \sigma^{\prime}=0-9-18-27 \mathrm{kPa} \\
& \tau=0-4.5-8.1-10.8 \mathrm{kPa}
\end{aligned}
$$

This envelope is plotted in the summary of results in Fig. 6.

For comparison a larger portion of the top part of the slope could be characterized by

$$
H=15 \mathrm{~m}, b=2.2, \gamma^{\prime}=10 \mathrm{kPa}
$$

leading to $p_{d}=p_{e}=150 \mathrm{kPa}$ for $r_{u}=0$

For $b=2.2$ one obtains, from Fig. 4

$$
\begin{aligned}
& r_{\sigma}=0-0.1-0.2-0.3 \\
& r_{\tau}=0-0.045-0.085-0.115
\end{aligned}
$$



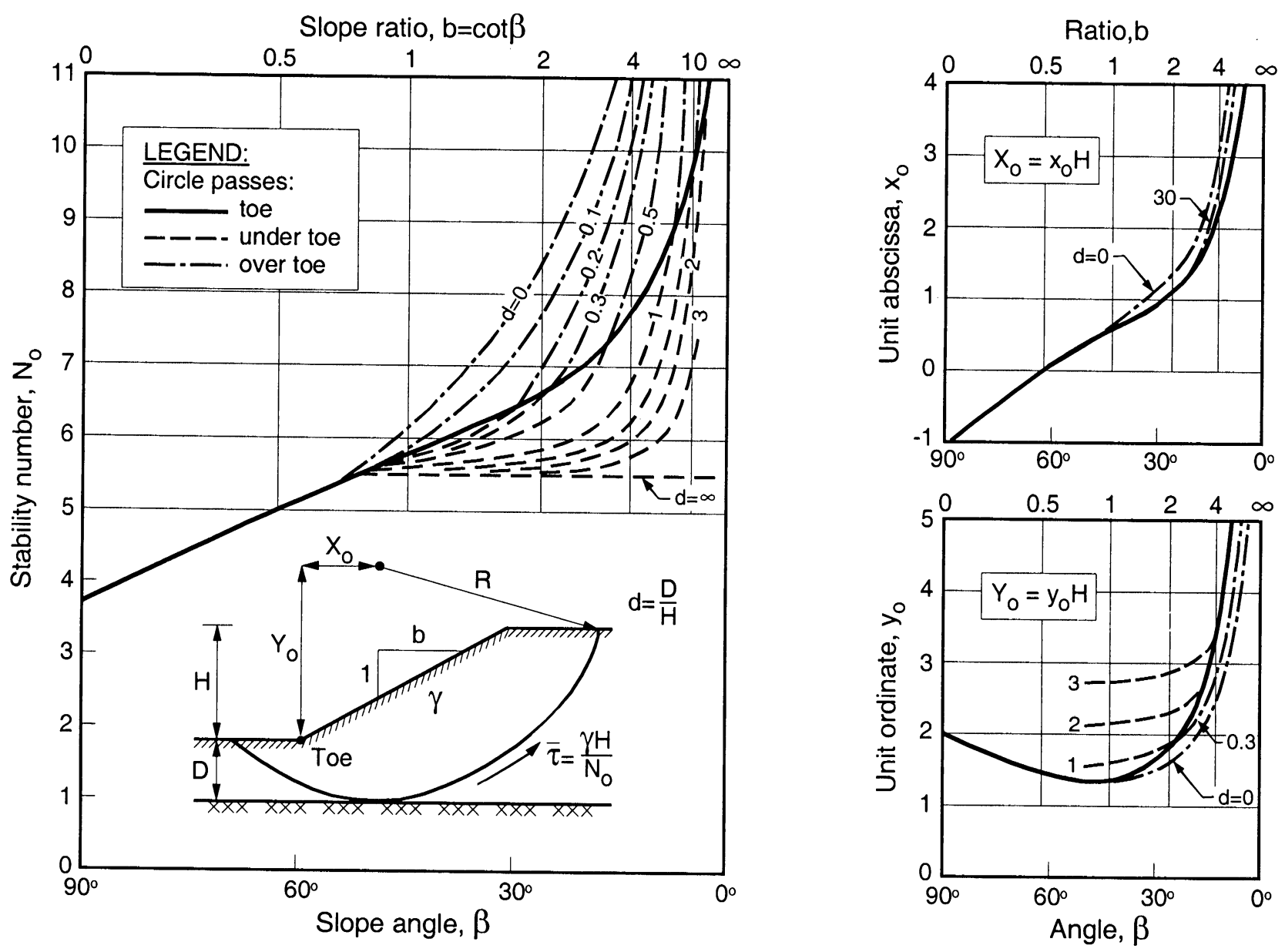

Fig. 5A Stability charts for total stress analysis
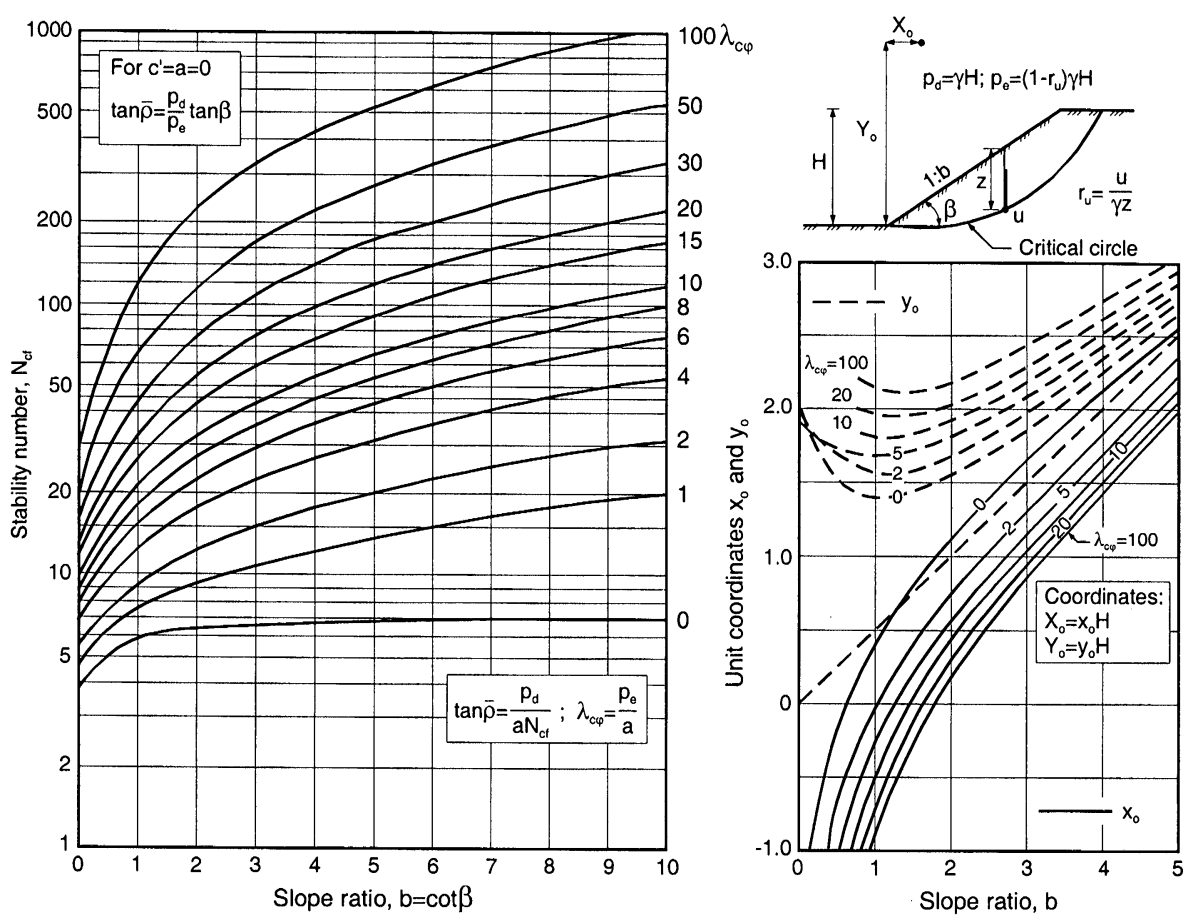

Fig. 5B Stability charts for effective stress analysis 


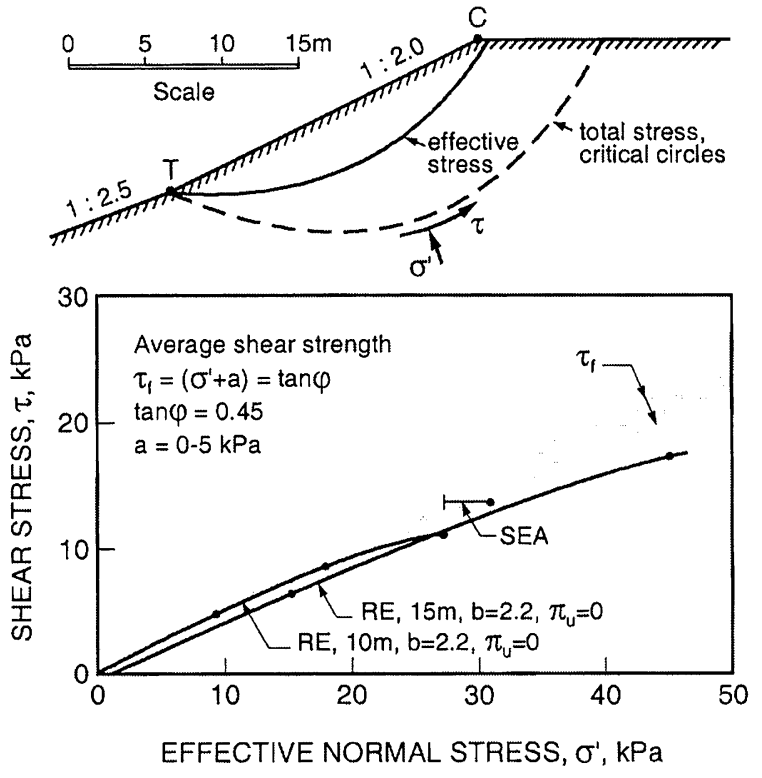

Fig. 6 Summary of results

leading to

$$
\begin{aligned}
& \sigma^{\prime}=0-14-30-45 \mathrm{kPa} \\
& \tau=0-6.8-12.7-17.2 \mathrm{kPa}
\end{aligned}
$$

This resistance envelope is also drawn in Fig. 6

Using the $S C$-diagram for total stress in Fig. $5 \mathrm{~A}$ one finds $N_{0}=6.7$ for $b=2$ as obtained before. Hence the same undrained, total stress result.

The dimensionless center coordinates $\left(x_{0} \sim 1,0\right)$ and $y_{0} \approx 1.75$ yield coordinates $X_{0}=10 \mathrm{~m}, Y_{0}=17,5 \mathrm{~m}$. This toe circle is drawn in Fig.6.

Using the SC-diagram for effective stress in Fig. $5 \mathrm{~B}$ one finds

$$
N_{c f}=45, x_{0}=0.35, y_{0}=2.0
$$

for $\lambda_{c \phi}=90 / 5=18$ and $b=2$.

Hence, for $a=5 \mathrm{kPa}$ and $p_{d}=90 \mathrm{kPa}$ one gets

$$
\tan \bar{\rho}=\frac{90}{5.45}=0.40 ; F_{e} \approx 1.1
$$

For $r_{u}=0.1, \lambda_{c \phi}=16.2$ and $N_{c f}=40$

$$
\tan \bar{\rho}=\frac{90}{5.40}=0.45, \quad F_{c}=1.0
$$

The center coordinates become

$$
X_{0}=3.5 \mathrm{~m}, Y_{0}=20 \mathrm{~m}
$$

The toe circle is drawn in Fig. 6.

As usual the effective stress analyses lead to a more shallow critical shear surface than the total stress analyses, based on a constant average, $s_{u}$.

The basic principle and the various numerical procedures which have been used so far for the slope at Finneidfjord are more than 45 years old. The results demonstrate very clearly that the numerical differences between the various simple approaches are of no consequence in practice, mainly because the uncertainties in shear strength are very much larger.

However, the simple classical approaches have two main limitations, giving average limit stresses only, and assuming regular slopes and circular shear surfaces, in plane strain.

\section{GENERALIZED PROCEDURE OF SLICES, GPS}

The first attempts to develop practical procedures for estimating the state of equilibrium stresses behind slopes were made in the midfifties, using limit equilibrium procedures on soil bodies defined by the slope and a shear surface of arbitrary shape through the soil profile.

The soil body is divided into a finite number of slices by vertical sections. One such slice is shown in detail in Fig. 7.

a)

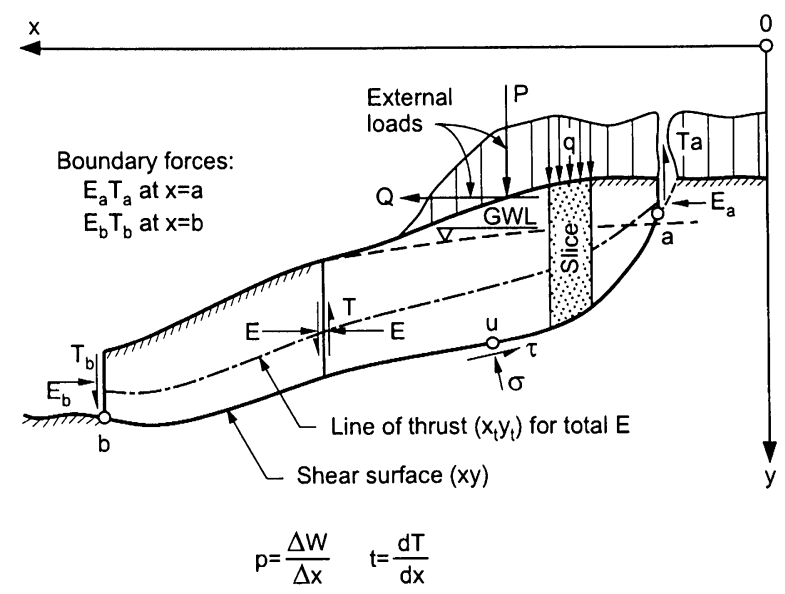

b)

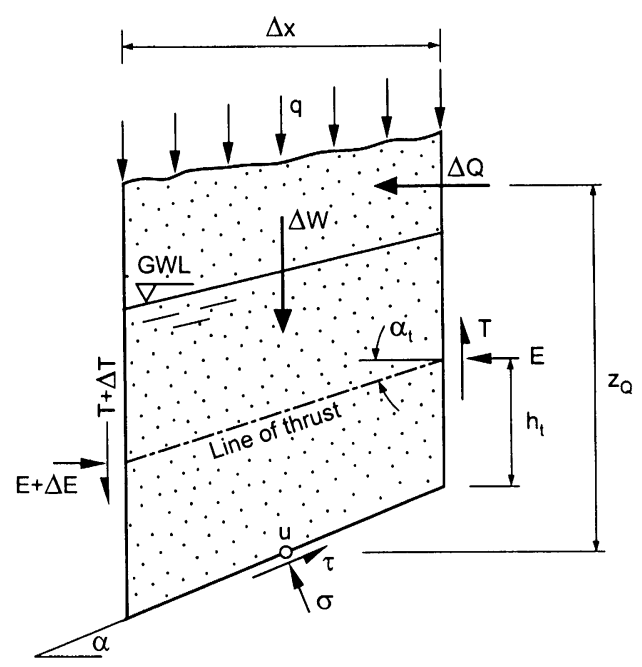

Fig. 7 Key sketches for developing the state of limit equilibrium stress for non-circular shear surfaces 
The vertical and horizontal equilibrium for each slice and for the whole body was established together with the moment equilibrium. Thus one obtained equilibrium values of the stresses $\left(\sigma^{\prime} \tau\right)$ along the selected surfaces, and the interslice forces $(E, T)$ on the vertical sections.

In 1957 the generalized procedure of slices, GPS was extended to earth pressure and bearing capacity analyses. Sliderule and/or mathematical tables handled the iteration procedure involved in the implicit set of equations.

A computer program for the GPS-procedure was first developed by Grande (1969). The derivations of all relevant equations and several examples of application of the computerized GPS are found in Janbu (1973).

The present paper will utilize directly the 1973 version of GPS, without repeating the derivations of formulas. All equations of the 1973 version have been rewritten in equilibrium form. The effective stress version is shown below.

The equilibrium approach aimed at finding the average equilibrium shear stress parameters

$$
\begin{array}{lr}
\tan \rho=f \cdot \tan \phi & \text { (effective) } \\
\tau_{c}=f \cdot s_{u} & \text { (total) }
\end{array}
$$

where $f=$ degree of shear mobilization in effective and or total stress.

A formula for $\tan \rho$ and or $\tau_{c}$ was obtained from the equation for overall horizontal equilibrium, $\Sigma \Delta E=0$. The combination of vertical and horizontal equilibrium for each slice had beforehand led to a general formula for $\Delta E$. Vertical equilibrium for each slice had given a simple formula for the normal stress $\sigma$, which combined with the equilibrium shear stress

$$
\tau=\left(\sigma^{\prime}+a\right) \tan \rho
$$

leads to the working formula for $\tau$. The equations for $\tan \rho, E, \tau$, and $\sigma$ were all statically indeterminate, containing the interslice parameter

$$
t=d T / d x
$$

where $T=$ total, vertical interslice force, see Fig. 7 .

The equation for moment equilibrium of a slice of infinitesimal width was found to be

$$
T=-E \tan \alpha_{t}+h_{t} \frac{d E}{d x}-z_{Q} \frac{d Q}{d x}
$$

where $h_{t}$ and $\tan \alpha_{t}$ define the line of total thrust.

This exact equation is the most unique feature of the GPS. With this equation the whole state of limit equilibrium can be solved easily by means of an itera- tion procedure, which today can be handled by a simple spread sheet analysis.

One first start by assuming $t=0$, from which one obtains a correct solution for $\tan \rho_{0}, E_{0}, \tau_{0}$ and $\sigma_{0}{ }^{\prime}$ corresponding to smooth interfaces $T=0$. With this solution one obtains a first approximation for $T=T_{1}$ and hence $t_{1}=d T_{1} / d x$ and thereby improved values for $\tan \rho, E$, $\tau_{1}$ and $\sigma_{1}{ }^{\prime}$ leading to $T_{2}$ and $t_{2}=d T_{2} / d x$ etc. The iteration usually converges rapidly.

The example in Fig. 8, dealing with the initial slide at Finneidfjord (top part of the slope) demonstrates the rapid convergence in $\tan \rho$ or $F$, and the final distribution of the interslice forces, $E$ and $T$, and the stresses $\tau$ and $\sigma^{\prime}$ along the shear surface. It is interesting to observe that there is also full mobilization $(\tan \rho \cong \tan \phi)$ along the interface between slices 4 and 5 . The values of $\tau$ and $\sigma^{\prime}$ on each slice are plotted in the $\tau-\sigma^{\prime}$ diagram in Fig. 8. It is seen to compare well with previous simple analyses, all of which indicate $F=f \sim 1.0$.

\section{CONCLUDING REMARKS}

This paper has shown that 50 years of development
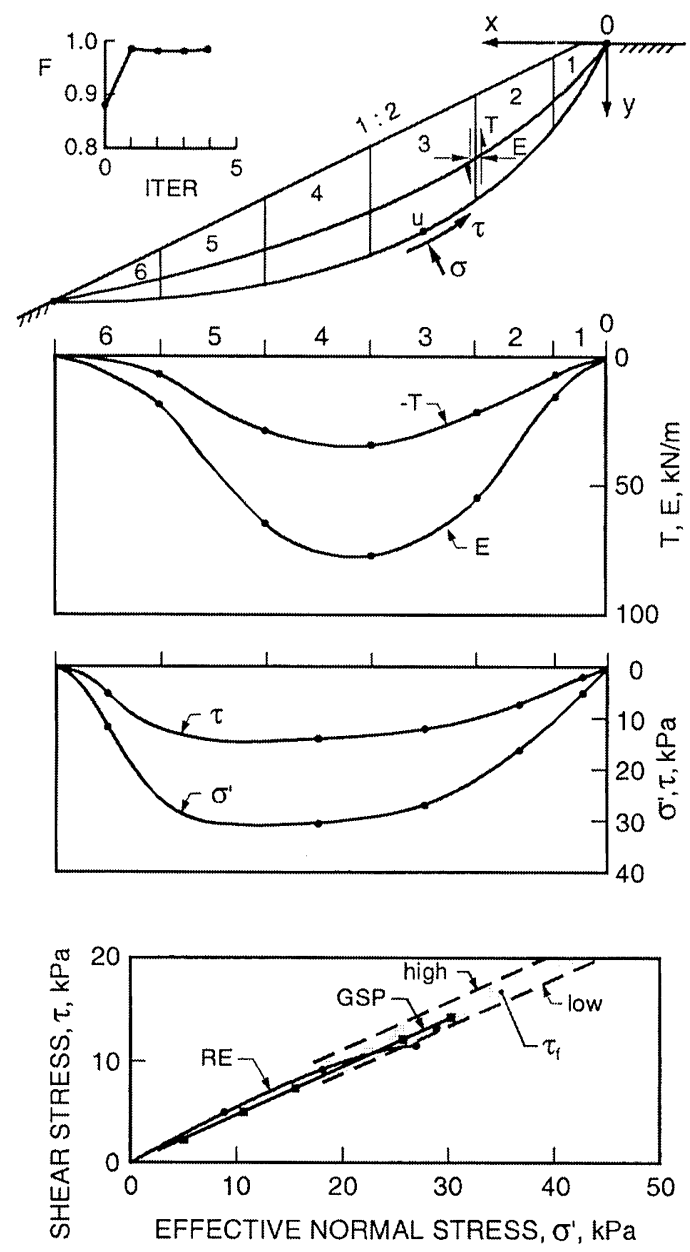

Fig. 8 Results of GPS-analysis 
in limit equilibrium analyses have led to both very simple (SEA, RE, SC) and more advanced (GPS) procedures for adequate solving of most conventional slope stability problems in practice. One of the important messages of this paper is that it is actually possible to determine the complete state of limit equilibrium behind slopes, independent of a priori knowledge of the shear strength.

The benefits are: simplicity, better understanding of the basics, and it becomes easier to identify the factors controlling slope stability. The procedures described above do not yield direct information about strain, and hence no movements.

However, todays advancement in realistic soil modelling coupled with high quality, computerized numerical procedures has made it possible to solve intricate deformation problems where coupled deformation, seepage and stability is dealt with. It is justified to look forward to the rich possibilities of further advancements in this field. Even so, the simpler procedures applied herein, will still be the best start, before more sophisticated procedures are applied.

\section{REFERENCES}

The applied references are, in order of their appearance in the text:

Hultin, S. (1916): "Grusfyllnader for kajbyggnader", Teknisk Tidsskrift, Vol. 31, Stockholm.

Pettersen, K. E. (1916) : “Kajraset i Göteborg den 5te mars 1916”, Teknisk Tidsskrift, Vol. 30 and Vol. 31, Stockholm.

Fellenius, W. (1918) : “Kaj- och jordraset i Göteborg”. Teknisk Tidsskrift, Vol. 2, Stockholm

Taylor, D. W. (1937): "Stability of Earth Dam Slopes”, Journal B. S. C. E, Vol. 24, No. 3, Boston.

Janbu, N. (1954): "Stability analysis of slopes with dimensionless parameters". Harvard Soil Mechanics Series, No. 46, 81 p. Cambridge, USA.

Janbu, N. (1957): "Earth Pressure and Bearing Capacity Calculations by Generalized Procedure of Slices". Proc. $4^{\text {th }}$ ICSMFE, London

Grande, L. (1969): "LAMMET-A Computer Program for Geotechnical Stability Analysis with Shear Surfaces of Any Shape". Soil mechanics, NTH, Norway (in Norwegian)

Janbu, N. (1973) : “Slope stability computations. The Embankment Dam Engineering”. Casagrande Volume, Editors Hirschfeld and Poulos. John Wiley \& Sons 1973, pp. 47-86. (The GPSprocedure covered in detail).

Länsivaara, T. (1995) : “Spreadsheet analyses of the GPS”. Internal report, Geoteknikk, NTH, Trondheim.

Janbu, N. (1996): "Slope stability evaluation in engineering practice". ISL, 1996, Trondheim.

(Received September 27, 2001, Accepted November 1, 2001) 